\title{
First exposure to rituximab is associated to high rate of anti-drug antibodies in systemic lupus erythematosus but not in ANCA-associated vasculitis
}

Francesca Faustini ${ }^{1,2^{*}}$ (D), Nicky Dunn ${ }^{3,4}$, Nastya Kharlamova ${ }^{3,4}$, Malin Ryner ${ }^{3,4}$, Annette Bruchfeld ${ }^{5,6}$, Vivianne Malmström ${ }^{1,4}$, Anna Fogdell-Hahn ${ }^{3,4+}$ and Iva Gunnarsson ${ }^{1,2+}$

\begin{abstract}
Background: Anti-drug antibodies (ADAs) can impact on the efficacy and safety of biologicals, today used to treat several chronic inflammatory conditions. Specific patient groups may be more prone to develop ADAs. Rituximab is routinely used for ANCA-associated vasculitis (AAV) and as off-label therapy for systemic lupus erythematosus (SLE), but data on occurrence and predisposing factors to ADAs in these diseases is limited.

Objectives: To elucidate the rate of occurrence, and risk factors for ADAs against rituximab in SLE and AAV.

Methods: $A D A s$ were detected using a bridging electrochemiluminescent (ECL) immunoassay in sera from rituximab-naïve (AAV; $n=41$ and SLE; $n=62$ ) and rituximab-treated (AAV; $n=22$ and $S L E ; n=66$ ) patients. Clinical data was retrieved from medical records. Disease activity was estimated by the SLE Disease Activity Index-2000 (SLEDAI-2 K) and the Birmingham Vasculitis Activity Score (BVAS).
\end{abstract}

Results: After first rituximab cycle, no AAV patients were ADA-positive compared to $37.8 \%$ of the SLE patients. Samples were obtained at a median (IQR) time of 5.5 (3.7-7.0) months (AAV), and 6.0 (5.0-7.0) months (SLE). ADA-positive SLE individuals were younger (34.0 (25.9-40.8) vs 44.3 (32.7-56.3) years, $p=0.002)$ and with more active disease (SLEDAl-2 K $14.0(10.0-18.5)$ vs. $8.0(6.0-14), p=0.0017)$ and shorter disease duration (4.14 (1.18-10.08) vs 9.19 (5.71-16.93), $p=0.0097)$ compared to ADA-negative SLE. ADAs primarily occurred in nephritis patients, were associated with anti-dsDNA positivity but were not influenced by concomitant use of corticosteroids, cyclophosphamide or previous treatments.

Despite overall reduction of SLEDAI-2 K (12.0 (7.0-16) to 4.0 (2.0-6.7), $p<0.0001)$, ADA-positive individuals still had higher SLEDAI-2 K (6.0 (4.0-9.0) vs $4.0(2.0-6.0), p=0.004)$ and their B cell count at 6 months follow-up was higher (CD19+\% 4.0 (0.5-10.0) vs $0.5(0.4-1.0), p=0.002)$. At retreatment, two ADA-positive SLE patients developed serum sickness (16.7\%), and three had infusion reactions (25\%) in contrast with one (5.2\%) serum sickness in the ADA-negative group.

\footnotetext{
${ }^{*}$ Correspondence: Francesca.Faustini@ki.se

${ }^{\dagger}$ Anna Fogdell-Hahn and Iva Gunnarsson shared last authorship.

${ }^{2}$ Unit of Rheumatology, Karolinska University Hospital, Stockholm, Sweden

Full list of author information is available at the end of the article
} permits use, sharing, adaptation, distribution and reproduction in any medium or format, as long as you give appropriate credit to the original author(s) and the source, provide a link to the Creative Commons licence, and indicate if changes were made. The images or other third party material in this article are included in the article's Creative Commons licence, unless indicated otherwise in a credit line to the material. If material is not included in the article's Creative Commons licence and your intended use is not permitted by statutory regulation or exceeds the permitted use, you will need to obtain permission directly from the copyright holder. To view a copy of this licence, visit http://creativecommons.org/licenses/by/4.0/. The Creative Commons Public Domain Dedication waiver (http://creativeco mmons.org/publicdomain/zero/1.0/) applies to the data made available in this article, unless otherwise stated in a credit line to the data. 
Conclusions: In contrast to AAV, ADAs were highly prevalent among rituximab-treated SLE patients already after the first course of treatment and were found to effect on both clinical and immunological responses. The high frequency in SLE may warrant implementations of ADA screening before retreatment and survey of immediate and late-onset infusion reactions.

Keywords: Rituximab, Anti-drug antibodies (ADA), Systemic lupus erythematosus, ANCA-associated vasculitis, Disease activity, B cell counts, ADA-screening

\section{Introduction}

Since the introduction of the chimeric monoclonal antiCD20 antibody rituximab (RTX) in the management of haematological malignancies [1], its use has been extended to several immune-mediated diseases including rheumatoid arthritis (RA) [2], anti-neutrophil cytoplasmic antibody (ANCA)-associated vasculitis (AAV) [3], systemic lupus erythematosus (SLE) [4] and multiple sclerosis (MS) [5]. In SLE, the drug is used as off label therapeutic, after two large randomized clinical trials having failed to meet their primary endpoints $[6,7]$. Nevertheless, there is a substantial body of literature that supports its use in SLE, especially as add-on medication in patients with refractory organ manifestations, mainly in lupus nephritis $[8,9]$.

The development of anti-drug antibodies (ADAs) is the result of an unwanted immune response which can occur against all biologicals including RTX and can impair the efficacy and safety of the treatment. The issues related to the potential immunogenicity of the biologics were recognized early in the process of their development. As a result, several efforts have been performed at a preclinical level to reduce the immunogenic potential of the different molecules. The primary reason for the biologics to be immunogenic resides in the proteinaceous nature of these molecules, their structure and the post-translational changes acquired in the production process [10].

RTX, as a chimeric antibody, can potentially carry in its mouse-derived variable regions several immunogenic epitopes that may elicit immune response in the individuals receiving the drug [11]. As a consequence, ADAs can recognize the target-binding domain of the molecule (i.e. recognize the idiotype), inhibiting the action of RTX. In addition, ADAs can also bind to other epitopes in the molecular structure of the drug, and through the formation of immune complexes, they can alter the pharmacokinetics through accelerated drug clearance, which in turn can reduce the clinical efficacy of the drug [10] or lead to infusion reactions.

In addition to the type of monoclonal antibody, the rate of occurrence of ADAs can also depend on other patientor drug-related factors, such as the route and frequency of administration of the drug, the different dosing regimens, the presence of co-medications and the underlying disease. Indeed, some systemic inflammatory diseases appear more prone to the development of ADAs than other illnesses [12].

In this respect, there is data which suggests that in RA, the occurrence of ADAs to RTX is affecting $4-11 \%$ of treated patients [2]. In contrast, in patients with relapsing-remitting MS, the proportion of patients developing ADAs to RTX has been shown to be as high as $37 \%$ and high titres of ADAs were associated with less profound $B$ cell depletion or earlier B cell repopulation [13].

In SLE, data concerning the rate of development of ADAs against the drug is still limited. From the clinical trials that have been carried out, SLE appears to be associated with both high rates of ADAs and high titres [4, 6 , 7] but their clinical relevance remains unclear. Longitudinal studies suggest that despite an initial response to RTX, ADA development may be a significant determinant of a secondary loss of efficacy at retreatment [14]. Alternatively, ADAs may be predictive of the occurrence of infusion reactions at retreatment, as shown by a recent observational study [15]. Studies addressing both the prevalence and impact of ADAs to RTX in SLE are still few and further research is needed.

Similarly, there is limited data available clarifying the impact of RTX immunogenicity in the context of AAV. To date, immunogenicity has only been evaluated in a small prospective study enrolling eleven AAV patients [16] and in a randomized clinical trial (RCT) in which $23 \%$ of the patients were found positive for ADAs within 18 months from treatment initiation, although the clinical implications of immunogenicity were unclear [3].

Further studies in a real-world setting are needed to confirm these findings and to better understand the clinical implications of ADA development, their impact on the safety profile of RTX and on its efficacy in systemic diseases such as SLE and AAV. Therefore, the aims of the present study were to elucidate the frequency of ADAs against RTX in two cohorts, one of SLE and one of AAV patients and, if ADA impact the intended pharmacodynamic effect of RTX in reducing the B cell count.

Secondary aims of this study were to identify risk factors for ADA development in these cohorts and to evaluate the association of ADAs with adverse events at retreatment in a clinical setting. Finally, we wanted to 
explore, if the development of ADAs was associated with subsequent lack of response on retreatment.

\section{Methods \\ Patients \\ SLE patients}

SLE patients included in the study belong to a clinical cohort prospectively enrolled at the Rheumatology Clinic at Karolinska University Hospital, Stockholm. All patients fulfilled the 1982 ACR criteria for SLE [17], were newly initiated on RTX and had a pre-treatment (baseline) sample, and at least one post treatment sample available (preferably at 6 months after first infusion), obtained at RTX trough prior to any subsequent infusions. A total of 66 SLE patients met the inclusion criteria and were included in the study.

\section{AAV patients}

AAV patients diagnosed and classified according to the EMA (European Medicines Agency) algorithm [18] were recruited from a larger cohort study which has been previously described [19].

In this cohort, patients were evaluated, and blood samples were collected at inclusion and at a scheduled 6 months follow-up visit. We retrospectively identified those, among the included patients who had received RTX, either at inclusion or within the 6 months followup of the cohort study design, and who had available samples taken before (baseline) and after treatment initiation. Since only 22 AAV patients had been treated with RTX, we also included additional samples from 19 AAV patients unexposed to RTX.

All patients provided informed consent prior to enrolment in the cohorts. This study was approved by the Stockholm ethics committee and conducted in accordance with the Helsinki declaration.

\section{Clinical data}

Clinical and demographic data were retrieved from the electronic clinical charts. For all patients, data on age, gender, disease duration, main indication for RTX administration, dose and treatment schedule and associated treatments was collected. For SLE patients, concomitant treatment at RTX initiation and previous treatments including corticosteroids (CS) and anti-rheumatic disease-modifying drugs (DMARDs) were included in the data collection. Disease activity was measured using the SLE Disease Activity Index-2000 (SLEDAI-2 K) [20]. For AAV patients, the Birmingham Vasculitis Activity Score (BVAS) was used [21].

\section{Rituximab administration}

In both cohorts, original RTX (Mabthera ${ }^{\circledR}$ ) was administered primarily using one of two different regimens including the haematological approved dosage of $375 \mathrm{mg} / \mathrm{m}^{2}$ of body surface area (BSA) every week over 4 weeks in accordance to previously published studies for both SLE [22] and AAV [3], or the RA approved dosage of $1 \mathrm{~g} 2$ weeks apart. In select cases, lower doses of $500 \mathrm{mg}$ 2 weeks apart were also used. Also, patients could receive concomitant intravenous cyclophosphamide, either as two infusions of $750 \mathrm{mg}$ or according to Euro-Lupus [23] or NIH (National Institutes of Health) protocol [24]. The addition of 6-metylprednisolone pulse therapy of 500$1000 \mathrm{mg}$ daily over 3 days was decided by the treating physician according to clinical needs.

\section{Laboratory investigations}

Baseline and 6 months follow-up laboratory measurements including total leucocytes, total lymphocytes and lymphocytes subpopulations of CD19+ cells were retrieved from clinical records where available. These measurements were conducted according to clinical routine. B cell depletion was defined as a total CD19+ positive cell count of $<0.01$ cells $/ \mu \mathrm{L}$ and a percentage of CD19+positive cells $<0.5$ out of total lymphocytes according to laboratory routine.

Additional laboratory measurements included in the dataset were complement levels ( $\mathrm{C} 3$ and $\mathrm{C} 4$ fractions) and serum anti-dsDNA titres. These were performed using different methods over the years, thus with different normal ranges. Therefore, these results were recorded as a categorical variable in the dataset (complement nor$\mathrm{mal} /$ low, anti-dsDNA positive/negative).

All laboratory analyses were performed at the Clinical Chemistry and Clinical Immunology Laboratory at the Karolinska University Hospital.

\section{Evaluation of renal response}

For SLE patients treated for lupus nephritis as the main indication and subsequently retreated, data on proteinuria (assessed as $24 \mathrm{~h}$ proteinuria (grams/24 h) or as urinealbumin to creatinine ratio, U-ACR, expressed in $\mathrm{mg} /$ $\mathrm{mmol})$ and creatinine levels $(\mathrm{mmol} / \mathrm{L})$ were collected in order to assess renal response after retreatment ( 6 and 12 months, respectively), according to commonly used definitions [25].

\section{Adverse events}

Clinical charts were reviewed for the occurrence of adverse events including infusion reactions (IR) at time of RTX retreatment. IR were defined according to their 
severity and modality to be handled in accordance with the Common Terminology Criteria for Adverse Events (CTCAE, v. 5).

Serum sickness was defined as a late-onset type III hypersensitivity reaction, characterized by the occurrence of fever, cutaneous rash and/or arthralgia/arthritis which usually occurs within 5-10 days after exposure to RTX [26, 27].

\section{Detection of ADA}

The presence of ADA against RTX was determined with an in-house validated bridging electrochemiluminescent (ECL) immunoassay, using the Meso Scale Discovery ${ }^{\circledR}$ (MSD) platform as previously described [13].

RTX-naïve samples (SLE; $N=62$, four samples excluded due to insufficient volume of sera available; and AAV; $N=41$ ) were used to establish SLE and AAV specific screening, confirmatory and titration cut-points for the immunoassay and to screen for naturally occurring ADA at baseline.

Follow-up samples (SLE; $N=66, \mathrm{AAV} ; N=22$ ) were used to evaluate for ADA development after RTX exposure. In brief, ADAs were detected through a three-tiered testing approach. Samples detected as positive for ADAs in the screening assay, using the disease-specific assay cut points, were subsequently assessed in a competitive assay to confirm the specificity of ADAs against RTX. The third step included titration of confirmed positive samples to determine ADA levels. The final titre was expressed as arbitrary units per millilitre $(\mathrm{AU} / \mathrm{mL})$. Confirmed positive samples below the titration cut point were given a titre of $<2 \mathrm{AU} / \mathrm{mL}$.

\section{Data analysis}

The primary outcome measures were ADA status (positive/negative) and titre (AU/mL) of a patient follow-up sample as determined using the bridging ECL assay.

A cut-off for lower ADA titres (lower or equal to 4 $\mathrm{AU} / \mathrm{mL}$ ) and higher ADA titres (above $5 \mathrm{AU} / \mathrm{mL}$ ) was adopted in accordance with previous studies [13].

In brief, in descriptive analysis, categorical variables are presented as numbers and percentages, and continuous variables are described as median and interquartile range (IQR). A univariate analysis was conducted to ascertain the relationship between ADA development and baseline characteristics. Similarly, 6-month follow-up measurements of CD19+ cells (expressed as percentage out of total lymphocytes or absolute counts $\left(N \times 10^{\wedge} 9^{9} / \mathrm{L}\right)$ in the peripheral blood) were compared when available with respect to the primary outcome.

To investigate if the presence of ADA was associated with adverse events or infusion reactions, clinical data from SLE patients who were retreated with RTX were evaluated and compared with ADA status and category.

Correlations between continuous variables were explored through Spearman's test. For the analysis of the association between categorical variables, the Fisher's or chi-square test were used as appropriate. To compare differences between groups, central measures of continuous variables were compared using the Mann-Whitney test as appropriate. To compare between time points within groups, Wilcoxon signed-rank test was applied.

Statistical analysis was conducted using SPSS software version 27. To produce graphs and figures some statistical tests were repeated using GraphPad Prism version 8. $P$ values $<0.05$ were deemed as significant.

\section{Results}

Clinical characteristics of the enrolled patients

Patient demographics and clinical characteristics for both diseases are summarized in Table 1 , and additional clinical information on SLE patients is provided in Table 2.

\section{SLE patients}

The majority of SLE patients (Table 1) were females (90.9\%), with a median (IQR) age of 36.3 (29.8-50.3) years and a disease duration of $7.9(2.6-14.3)$ years at the time of RTX initiation. Their disease activity was moderate to high (SLEDAI-2 K median (IQR) 12.0 (7.0-16.0)), and the main indication for RTX use was lupus nephritis (63.6\%) (Table 2).

Table 1 Baseline characteristics of the RTX-treated SLE and AAV patients

\begin{tabular}{|c|c|c|}
\hline & $\operatorname{SLE}(n=66)$ & $\operatorname{AAV}(n=22)$ \\
\hline \multicolumn{3}{|l|}{ Clinical features } \\
\hline Females $(n,(\%))$ & $60(90.9)$ & $12(54.5)$ \\
\hline Age (median (IQR)) & $36.3(29.8-50.3)$ & $62.0(44.0-70.5)$ \\
\hline Disease duration (median (IQR)) & $7.9(2.6-14.3)$ & $1.5(0-6.5)$ \\
\hline \multicolumn{3}{|l|}{ Disease activity } \\
\hline SLEDAI-2K $(n=65)($ median $(\mathrm{QQR}))$ & $12.0(7.0-16.0)$ & - \\
\hline $\operatorname{BVAS}(n=17)($ median $(\mathrm{IQR}))$ & - & $15.0(10.5-22.5)$ \\
\hline \multicolumn{3}{|l|}{ Rituximab treatment regimens } \\
\hline $\begin{array}{l}375 \mathrm{mg} / \mathrm{m}^{2} \times 4 \text { weekly infusions } \\
(n, \%)\end{array}$ & $38(57.6)$ & $5(22.7)$ \\
\hline $1 \mathrm{~g} 2$ weeks apart $(n, \%)$ & $24(36.4)$ & $13(59.1)$ \\
\hline 500 mg 2 weeks apart $(n, \%)$ & $4(6.1)$ & $3(13.6)$ \\
\hline \multicolumn{3}{|l|}{ Concomitant treatments } \\
\hline IV cyclophosphamide ( $n, \%)$ & $36(54.5)$ & $10(45.4)$ \\
\hline IV 6-methylprednisolone $(n, \%)$ & $37(56.1)$ & $4(18.2)$ \\
\hline
\end{tabular}

IQR Interquartile range, SLEDAI- 2 K Systemic Lupus Erythematosus Disease Activity Index 2000, BVAS Birmingham Vasculitis Activity Index, IV Intravenous ${ }^{a}$ One patient (indication peripheral polyneuropathy) was excluded from the calculation 
Table 2 Specific clinical characteristics and pharmacological history of the SLE patients

\begin{tabular}{|c|c|}
\hline & SLE $(n=66)$ \\
\hline \multicolumn{2}{|l|}{ Baseline laboratory findings } \\
\hline Total leukocytes ( $N$ cells/mm³, median (IQR)) & $7.0(4.5-9.1)$ \\
\hline Total lymphocytes ( $N$ cells $/ \mathrm{mm}^{3}$, median (IQR)) & $0.9(0.5-1.4)$ \\
\hline CD19 positive cells $(n=37)\left(N\right.$ cells $/ \mathrm{mm}^{3} /$ percentage median (IQR)) & $0.05(0.03-0.12) / 7.0(4.0-13.0)$ \\
\hline Anti-dsDNA positivity, $(n, \%)$ & $44(66.7)$ \\
\hline Low complement levels $(n=61),(n, \%)$ & $35(57.4)$ \\
\hline \multicolumn{2}{|l|}{ Primary indication for RTX } \\
\hline Lupus nephritis, $n$ (\%) & $42(63.6)$ \\
\hline Neurolupus, $n(\%)$ & $7(10.6)$ \\
\hline Lupus related arthritis, $n(\%)$ & $7(10.6)$ \\
\hline Mucocutaneous manifestations, $n(\%)$ & $2(3.0)$ \\
\hline Haematological manifestations, $n(\%)$ & $5(7.6)$ \\
\hline Other manifestations ${ }^{\mathrm{a}}, \mathrm{n}(\%)$ & $3(4.5)$ \\
\hline \multicolumn{2}{|l|}{ Ongoing treatment at RTX initiation } \\
\hline Oral Prednisolone, $n(\%)$ & $61(92.4)$ \\
\hline Daily dose, mg (median (IQR)) & $12.5(12.5-20.0)$ \\
\hline Antimalarials, $n(\%)$ & $27(40.9)$ \\
\hline Azathioprine, $n(\%)$ & $6(9.1)$ \\
\hline Mycophenolate, $n(\%)$ & $9(13.6)$ \\
\hline Methotrexate, n (\%) & $3(4.5)$ \\
\hline \multicolumn{2}{|l|}{ Previous treatments } \\
\hline Cyclophosphamide, $n(\%)$ & $40(60.6)$ \\
\hline Cumulative dose (median (IQR)) & $6075(3150-9275)$ \\
\hline Azathioprine, $n(\%)$ & $44(66.7)$ \\
\hline Mycophenolate, n (\%) & $32(48.5)$ \\
\hline Methotrexate, $n(\%)$ & $17(25.8)$ \\
\hline Cyclosporine A, $n(\%)$ & $11(16.7)$ \\
\hline
\end{tabular}

IQR Interquartile range, $n$ Number, CD19 Cluster of differentiation 19, anti-dsDNA Anti-double strand-DNA antibodies

${ }^{a}$ Other manifestation: one fatigue and systemic inflammation, one lung fibrosis, one peripheral polyneuropathy

In both disease cohorts, RTX treatment had been administered according to different schedules. The majority of SLE patients (Table 1) were treated according to the haematological regimen (57.6\%). Moreover, more than half of the patients had received intravenous cyclophosphamide (54.5\%) and 6-metylprednisolone pulse therapy (56.1\%) concomitant to RTX. A total of 31 SLE patients were retreated with RTX, 11 of these within one year from their first infusion.

The majority of SLE patients (92.4\%) received oral Prednisolone at the time of RTX initiation; however, a majority did not receive concomitant DMARD treatment (72.7\%). At baseline, the prevalence of anti-dsDNA positivity was as high as $66.7 \%$, and complement activation was present in $57.4 \%$ of SLE patients (Table 2).

\section{AAV patients}

The AAV cohort $(n=22)$ had a median (IQR) age of 62.0 $(44.0-70.5)$ years and a disease duration of $1.5(0-6.5)$ years. In this cohort, RTX was primarily administered in a dosage of $1 \mathrm{~g} 2$ weeks apart (59.1\%) and a smaller proportion received intravenous cyclophosphamide (45.4\%) and 6-metylprednisolone pulses (18.2\%) as co-medication (Table 1).

The majority of AAV patients had a diagnosis of granulomatosis with polyangiitis (GPA, $n=15$ ) or microscopic polyangiitis (MPA, $n=6$ ) accounting for 68.2 and $27.3 \%$ of the cohort respectively. Only one patient (4.5\%) was included with eosinophilic granulomatosis with polyangiitis (EGPA).

\section{Frequency of ADAs in SLE patients}

A total of three patients $(4.8 \%, 3 / 62)$ had detectable $\mathrm{ADA}$ at baseline, but all with very low titres $(<2 \mathrm{AU} / \mathrm{ml})$. Of these three patients, one progressed at follow-up to develop higher titres of ADA (64 AU/mL) following the first RTX treatment course, one remained positive at 
very low titre $(<2 \mathrm{AU} / \mathrm{mL})$ and one converted to ADAnegative after treatment.

Follow-up samples from patients with SLE $(n=66)$ were obtained at a median (IQR) of 6.0 (5.0-7.0) months from the first RTX treatment cycle, and immediately prior to the next infusion, if continued treatment was given (five patients were retreated between 6 and 8 months from the first cycle).

ADAs were detected in $37.8 \%$ of SLE patients (25/66) at follow-up. Of the positive patients, 6 were positive at low titres (lower or equal to $4 \mathrm{AU} / \mathrm{mL}$ ), while 19 had higher titres (higher than $5 \mathrm{AU} / \mathrm{mL}$, range 8-4000).

\section{Frequency of ADA in the AAV patients}

At baseline, one AAV patient was detected as ADApositive but also with a low titre $(<2 \mathrm{AU} / \mathrm{ml})$ and no patient was detected ADA-positive at follow up $(n=22)$, including the patient positive at baseline. The median (IQR) time of follow-up between RTX initiation and follow-up sampling was 5.5 (3.7-7.0) months.

\section{Association of ADA status with baseline clinical} and immunological characteristics

The baseline clinical and immunological characteristics for ADA-positive and ADA-negative SLE patients are shown in Table 3.

In the univariate analysis (Table 3), ADA-positive SLE patients were significantly younger compared to ADAnegative (median (IQR) 34.0 (25.9-40.8) vs 44.3 (32.7$56.3)$ years, $p=0.002)$ and with shorter disease duration (median (IQR) 4.14 (1.18-10.08) vs 9.19 (5.71-16.93, $p=0.0097)$. ADA-positive patients also had a higher baseline disease activity (median (IQR) SLEDAI-2 K 14.0 (10.0-18.5) vs. 9.19 (5.71-16.93), $p=0.0017)$. Patients being treated for lupus nephritis had a higher rate of ADA compared to SLE patients treated for other indications $(22 / 42,(52.4 \%)$ vs $3 / 24,(15.5 \%)$, Fisher exact test, $p=0.001$, Fig. 1a).

Baseline anti-dsDNA positivity was also significantly associated with ADA positivity at follow-up with $47.7 \%$ (21/44) of anti-dsDNA-positive patients baseline developing ADA compared to $18.2 \%(4 / 22)$ of the ADAnegative patients (Fisher's exact test $p=0.03$, Fig. 1b).

Table 3 Comparison of the baseline characteristics of ADA-positive and ADA-negative SLE patients

\begin{tabular}{|c|c|c|c|}
\hline & ADA positive $(n=25)$ & ADA negative $(n=41)$ & $P$ value* \\
\hline \multicolumn{4}{|l|}{ Patient characteristics } \\
\hline Age (years, median (IQR)) & $34.0(25.9-40.8)$ & $44.3(32.7-56.3)$ & 0.002 \\
\hline Sex (Females, $n, \%)$ & $27(87.1)$ & $33(94.2)$ & 0.66 \\
\hline Disease duration (years, median (IQR)) & $4.14(1.18-10.08)$ & $9.19(5.71-16.93)$ & 0.0097 \\
\hline \multicolumn{4}{|l|}{ Disease activity } \\
\hline SLEDAI-2 K (median (IQR)) & $14.0(10.0-18.5)$ & $8.0(6.0-14.0)$ & 0.017 \\
\hline \multicolumn{4}{|l|}{ Laboratory data } \\
\hline Total leukocytes ( $\mathrm{N}$ cells/mm³ median (IQR)) & $6.3(4.1-8.1)$ & $7.1(4.7-9.5)$ & 0.53 \\
\hline Total lymphocytes ( $N$ cells $/ \mathrm{mm}^{3}$ median (IQR)) & $0.98(0.54-1.47)$ & $0.85(0.5-1.52)$ & 0.74 \\
\hline CD19+ cells (\%, median (IQR)) & $8.0(5.25-14.5)$ & $6.0(2.5-11.5)$ & 0.17 \\
\hline CD19+cells $(N \times 10 \wedge 9 / L$, median $(I Q R))$ & $0.065(0.03-0.15)$ & $0.05(0.03-0.085)$ & 0.21 \\
\hline \multicolumn{4}{|l|}{ Treatments } \\
\hline Concomitant CS pulses, \% ( $n$ ) & $60.0(15)$ & $53.6(22)$ & 0.61 \\
\hline Concomitant cyclophosphamide, \% (n) & $56.0(14)$ & $53.6(22)$ & 0.85 \\
\hline Cumulative dose of concomitant cyclophosphamide (mg, (median (IQR)) & $1200(950-1600)$ & $1000(1000-1600)$ & 0.81 \\
\hline Previous cyclophosphamide, \% (n) & $60.0(15)$ & $60.0(25)$ & 0.93 \\
\hline Cumulative dose of previous cyclophosphamide (mg, median (IQR)) & $6700(4500-10000)$ & 6000 (3000-9150) & 0.58 \\
\hline Corticosteroids at baseline, \% (n) & $92(23)$ & $92.6(38)$ & 0.91 \\
\hline Total corticosteroid dose at baseline (mg, median (IQR)) & $12.5(10-17.5)$ & $15.0(7.5-20.0)$ & 0.73 \\
\hline Antimalarial treatment at baseline, $\%(n)$ & $36.0(9)$ & $43.9(18)$ & 0.53 \\
\hline DMARDs at baseline (AZA, MMF, MTX), n & $0 / 4 / 1$ & $6 / 5 / 2$ & $\mathrm{n} / \mathrm{a}$ \\
\hline \multicolumn{4}{|l|}{ Rituximab treatment (from baseline) } \\
\hline Schedule of RTX $\left.\left(n, 375 \mathrm{mg} / \mathrm{m}^{2} \times 4,1 \mathrm{~g} \times 2,500 \mathrm{mg} \times 2\right)\right)$ & $14 / 10 / 1$ & $24 / 14 / 3$ & 1.00 \\
\hline Cumulative dose of RTX at first course (mg, median (IQR)) & $2100(2000-2650)$ & $2400(2000-2800)$ & 0.33 \\
\hline
\end{tabular}

IQR Interquartile range, SLEDAI-2 K SLE Disease Activity Index-2000, CD19 Cluster of differentiation 19, RTX Rituximab, n/a Not applicable, DMARDs Disease-modifying anti-rheumatic drugs, AZA Azathioprine, MMF Mycophenolate mofetil, MTX Methotrexate, CS Corticosteroids

* Mann-Whitney test 


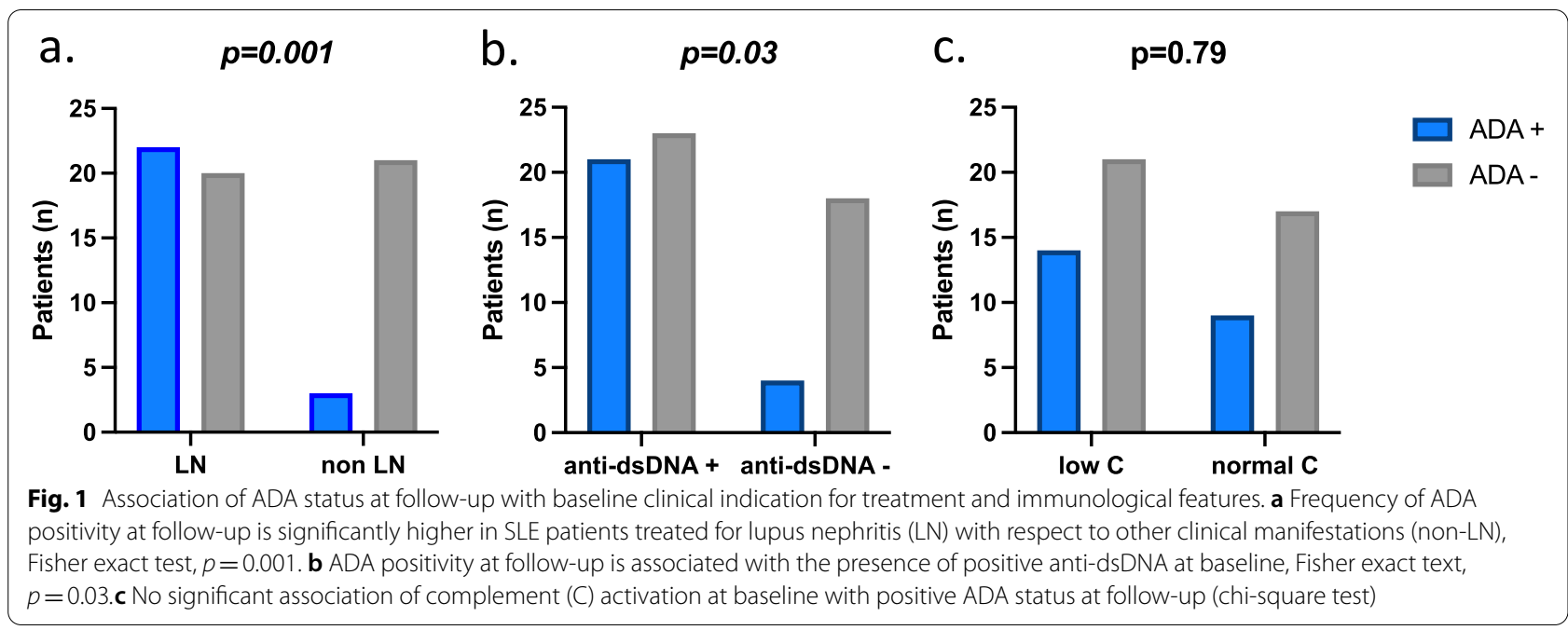

Conversely, the anti-dsDNA positive patients were also more prevalently affected by lupus nephritis $(n=32$, $72.7 \%)$.

No difference was seen in ADA development between groups regarding RTX treatment schedule $(p=1.00)$ or cumulative dose $(p=0.33)$. Furthermore, no difference was seen between ADA-positive and negative groups in previous exposure and concomitant treatment with intravenous cyclophosphamide (chi-square test, $p=0.93$ and $p=0.58$, respectively) or intravenous corticosteroid pulse therapy at time of RTX infusion (chi-square test, $p=0.91)$. Similarly, the use of antimalarials, oral corticosteroids or DMARDs prior to RTX was not associated with the presence of ADAs at follow-up (Table 3). Finally, no association was seen between ADA and baseline complement activation (chi-squared test, $p=0.79$, Fig. 1c).

\section{Association of ADA development and peripheral B cell depletion}

Data on peripheral blood lymphocytes subpopulations as measured by flow cytometry were available for 37 of the SLE patients at baseline and 37 patients at 6 months of follow-up.

No statistically significant differences were found regarding the baseline $\mathrm{B}$ cell count between ADA-positive and negative SLE patients at follow-up.

At 6 months follow-up, a higher proportion of CD19+to total lymphocytes was seen in ADA-positive patients compared to ADA-negative patients (median (IQR) $4.0(0.5-10.0)$ vs $0.5(0.4-1.0), p=0.002)$ (Fig. 2a). Similarly, when comparing the absolute count of CD19+ cells in the peripheral blood, significant differences were observed, with a median (IQR) 0.03 $(0.01-0.14)$ vs $0.01(0.01-0.01), p=0.003)$ comparing ADA-positive and ADA-negative patient groups (Fig. 2b).
A higher proportion of patients with ADA-positive status at follow-up did not achieve the status of B cell depletion (CD19\% in peripheral blood $<0.5 \%$ ), although this did not reach statistical significance (Fig. 2d-f).

No significant correlation was found between the ADA status and the proportion of patients who achieved B cell depletion (BCD) at follow-up (Fig. 2d). Thereafter, we stratified ADA-positive patients by low titre (lower or equal to $4 \mathrm{AU} / \mathrm{ml}$ ), and high titres (higher than $5 \mathrm{AU} / \mathrm{ml}$ ).

No significant difference was seen between B cell depletion in low and high ADA-positive titre groups (Fig. 2e). Moreover, when comparing higher titre ADA-positive patients to negative ADA patients, it was not statistically significant (Fig. 2f).

\section{Adverse events associated with ADA at retreatment}

Thirty-one of the 66 SLE patients underwent retreatment over time. Of these, 12 (38.7\%) were ADA-positive and retreated at a median of $17.5(10.2-66.7)$ months after the first infusion. The remaining 19 patients $(61.3 \%)$, who were ADA-negative, were retreated after a median of 19.0 (9.7-67.0) months following the first RTX infusion.

Of the 12 ADA-positive SLE patients who were retreated, three $(25.0 \%, 3 / 12)$ had immediate infusion reactions and two (16.7\%) manifested serum sickness following the second course of RTX treatment. Of the three patients with immediate infusion reactions two had an ADA titre of $80 \mathrm{AU} / \mathrm{mL}$ and one a titre of $8 \mathrm{AU} / \mathrm{mL}$ and were retreated at 10, 11 and 115 months from the first course of RTX respectively. The two SLE patients who developed serum sickness had an ADA titre of $4000 \mathrm{AU} / \mathrm{mL}$ and $4 \mathrm{AU} / \mathrm{mL}$ respectively and retreatment was administered at 8 and 23 months respectively from the first course of RTX. There were no immediate infusion reactions in ADA-negative patients at retreatment 


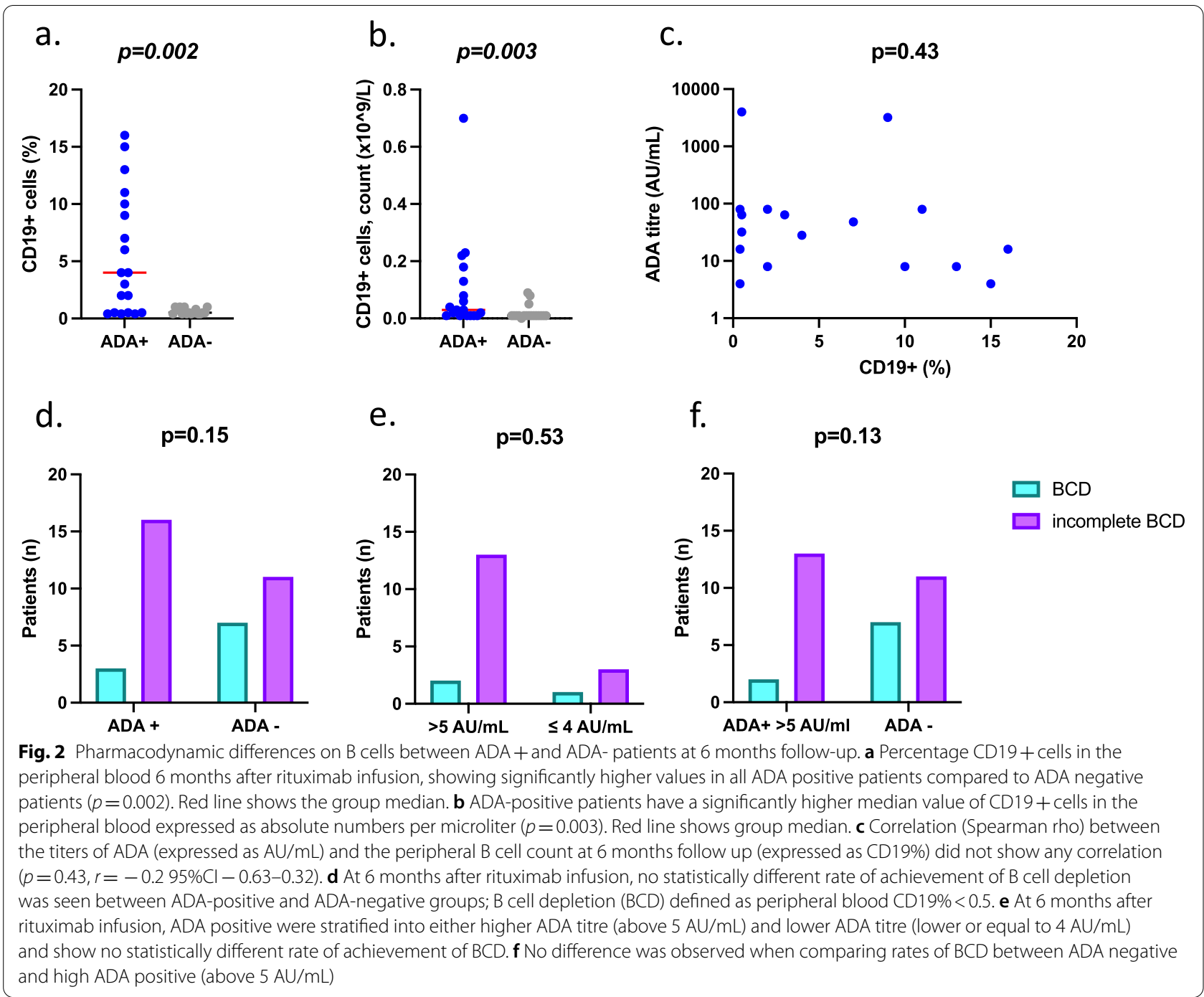

however, one patient presented with a late-onset reaction suggestive of serum-sickness $(5.2 \%, 1 / 19)$.

\section{Variations in disease activity (SLEDAI-2 K) after rituximab treatment}

Overall, the entire group of SLE patients responded with a reduction of median SLEDAI- $2 \mathrm{~K}$ of eight units, from $12.0(7.0-16.0)$ at baseline to $4.0(2.0-6.7)$ at 6 months follow-up (Wilcoxon signed-rank test $p<0.0001$ ). A significant reduction of disease activity was observed both in ADA-positive and ADA-negative patients $(p<0.0001$ in each subgroup). However, the ADA-positive patients still had a higher disease activity at 6 months follow-up compared to ADA-negative patients (Mann-Whitney test $p=0.004)$. Stratifying by indication to RTX initiation, a reduction of disease activity was evident both for SLE patients treated for lupus nephritis and for those treated for all other indications (Wilcoxon signed-rank test $p<0.0001$ for both subgroups).

In the lupus nephritis group, the patients ADA-positive at follow-up, showed a significant reduction of the SLEDAI-2 K score from baseline to 6 months follow-up $(p<0.0001)$, similarly to ADA-negative lupus nephritis patients $(p<0.0001)$. However, their disease activity was higher at follow-up compared to the ADA-negative patients with lupus nephritis $(p=0.02)$ (Fig. 3).

\section{Efficacy of RTX after retreatment in lupus nephritis ADA-positive patients}

Among the 42 SLE patients who had been started on RTX for active lupus nephritis, a total of 17 had undergone retreatment at some point (median (IQR) time to retreatment 38.0 (11.5-81.0) months). Of these 17 patients, 9 were found to be ADA-positive 6 months after the first RTX course (before retreatment), with a median 


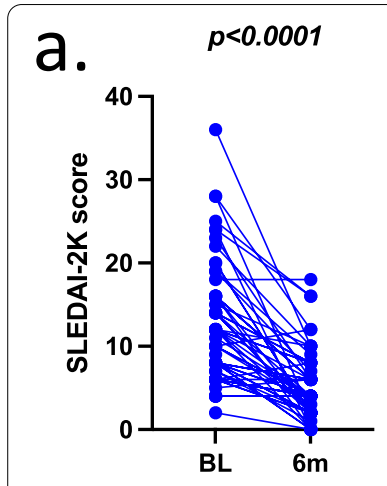

All SLE patients $(n=65)$

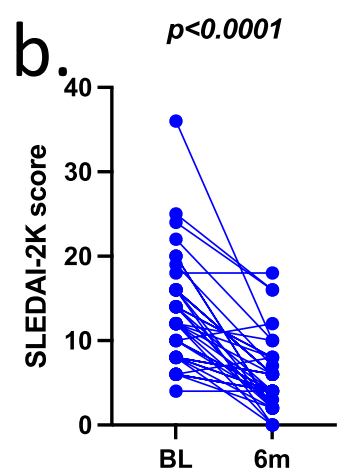

Lupus nephritis $(\mathrm{N}=42)$

C.

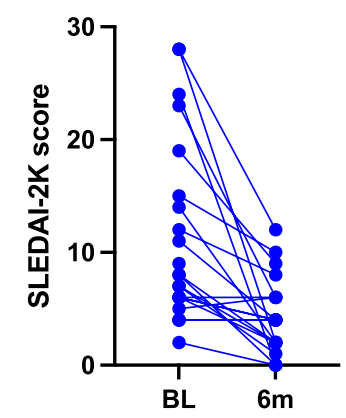

Non-lupus nephritis $\mathbf{(} \mathbf{N}=\mathbf{2 3})$

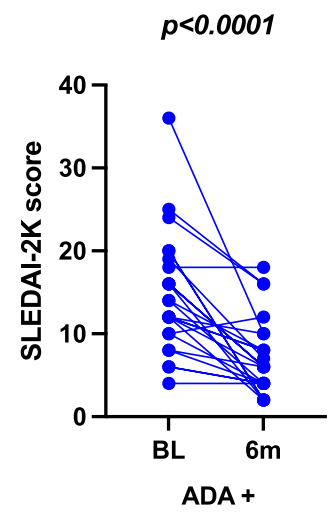

$p<0.0001$

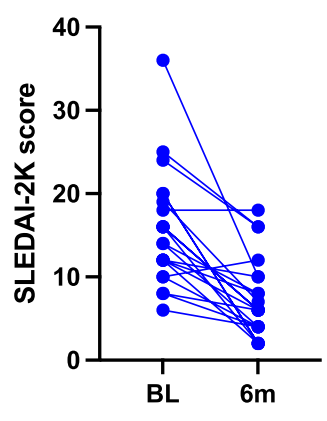

LN, ADA +

$p=n s$

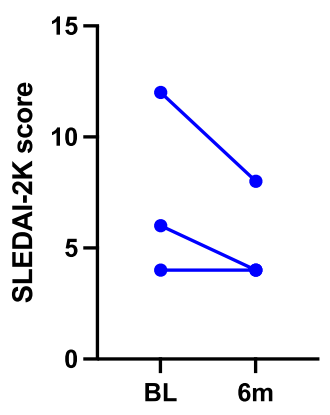

Non-LN, ADA +

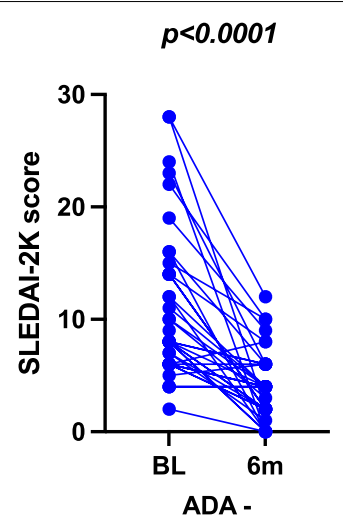

$p<0.0001$

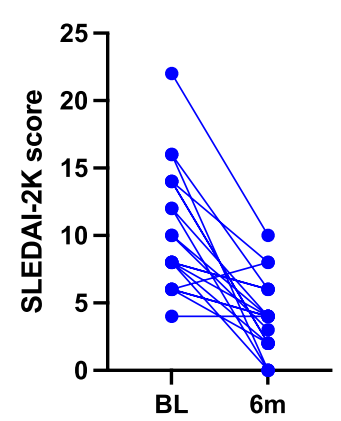

LN, ADA -

$p<0.0001$

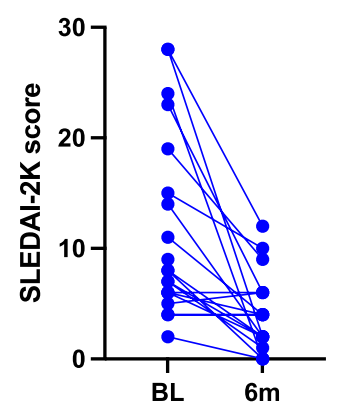

Non-LN, ADA -

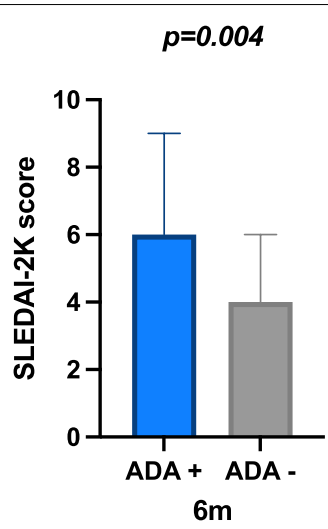

$p=0.02$

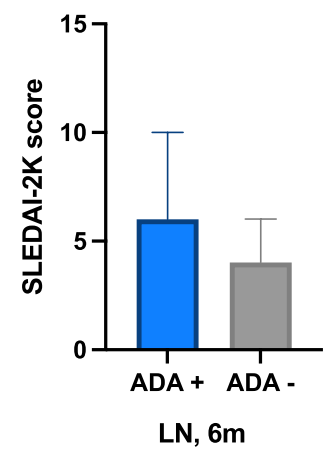

$p=0.37$

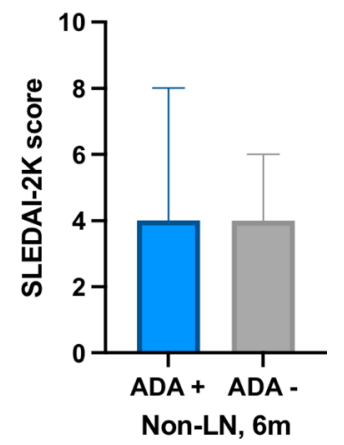

Fig. 3 Changes in disease activity in SLE patients, as measured by SLEDAI-2 K. a The SLEDAI-2 K score shows a significant reduction in the whole SLE cohort ( $N=65$, one value missing) from baseline to 6-months follow-up, with significant reduction that is confirmed also for patients ADA-positive and ADA-negative at 6-months follow-up (Wilcoxon signed-rank test, $p<0.0001$ ). When evaluated at 6-months follow-up, the ADA-positive patients showed however higher SLEDAI-2 K values compared to ADA-negative patients (Mann-Whitney test $p=0.004$ ). b Similar behaviour, with significant reduction of SLEDAI-2 K between baseline and 6-months follow-up, was observed for lupus nephritis (LN) patients and maintained whether they were ADA-positive or ADA negative at follow-up (Wilcoxon signed-rank test $p<0.0001$ for all). Also in LN patients, SLEDAl-2 K values at 6-months follow-up were higher in ADA-positive patients compared to ADA-negative ones (Mann-Whitney test, $p=0.02$ ). c Patients treated for clinical indications other than lupus nephritis (non-lupus nephritis, non-LN) also showed a significant reduction of the SLEDAl- $2 \mathrm{~K}$ at a group level and in both ADA-positive and ADA-negative subgroups (Wilcoxon signed-rank test $p<0.0001, p=\mathrm{ns}$ and $p<0.0001$, respectively). In these patients, SLEDAI- $2 \mathrm{~K}$ values, measured at 6-months, did not differ in a statistically meaningful manner, between ADA-positive and ADA-negative subgroups (Mann-Whitney test, $p=0.37$ ) 
(IQR) ADA titre of $48.0(6.0-80.0)$ AU/mL. Seven of these retreated lupus nephritis patients (7/17) had early retreatment, i.e. within 18 months from the nephritis flare for which RTX was started, two for non-nephritic flares (one ADA-positive and one ADA-negative) and five for nephritic flares (four of which ADA-positive), respectively.

Of the latter four ADA-positive patients who had a nephritic flare requiring early retreatment, three were non-responders and one partial responder at 6 and 12 months from retreatment [25]. All except for one patient with ADA titre $<2 \mathrm{AU} / \mathrm{mL}$, were found to have high titres of ADA (48, 64 and $4000 \mathrm{AU} / \mathrm{ml}$, respectively).

\section{Discussion}

In this study, we showed that RTX treatment is associated with a high rate of ADA positivity in SLE patients already after the first course of treatment. Moreover, ADA occurrence was not influenced by concomitant administration of intravenous corticosteroids or immunosuppressants such as cyclophosphamide. Among the SLE patients, ADAs were mainly observed in patients with nephritis and a serologically active profile with anti-dsDNA antibody positivity. In contrast, the development of ADAs was not observed in AAV patients after exposure to the first course of RTX.

The development of ADAs (in the previous literature also described as HACA, human-anti-chimeric antibodies) has been reported in early clinical trials [4] evaluating RTX in SLE. In this context, ADAs were more commonly observed in patients who exhibited higher disease activity at baseline and were of Afro-American ethnic background. In addition, when in high titres, they were associated to less efficient $\mathrm{B}$ cell depletion and lower RTX serum concentrations. Similarly, the two RCT $[6,7]$ evaluating RTX in SLE report on the presence of ADA (HACA), in $26.1 \%$ and $15.1 \%$ of the patients in the active treatment arm, with some patients experiencing adverse events. More recently, studies on real-life cohorts, have underscored how ADA can be implicated in the loss of clinical efficacy and less efficient B cell depletion of peripheral blood at retreatment in SLE patients [14].

In our study, we reported a frequency of $37.8 \%$ ADA positivity in SLE patients after the first exposure to RTX, consistent with the $37 \%$ reported by Wincup et al., who also used a bridging ECL immunoassay for ADA detection. Similarly, in line with their findings, our ADA-positive patients were younger at the first exposure to RTX compared to ADA-negative individuals [15]. This is in contrast with observations concerning ADAs against other biotherapies, such as interferon-beta in MS, where ADA-positive patients were significantly older than patients with persistently negative results for ADA testing [28].

Disease activity at baseline, was higher in ADA positive patients in our study, which contrasts to what was described by Wincup et al. [15], probably reflecting the fact that our cohort was more skewed towards a higher prevalence of severe lupus manifestations including active nephritis and CNS patients. This could also explain how baseline immunological features such as antidsDNA positivity were found to be associated to ADA positivity at follow-up in our study. Even though such features may differentiate our patients from other cohorts, it is relevant to highlight how the patients more prone to develop ADA in our SLE cohort, were those with refractory organ involvement such as lupus nephritis and positivity for anti-dsDNA. This observation is of major relevance, since it underlines how patients more in need of additional treatment, who are the usual candidates to RTX, are also those more susceptible to ADAs development. Also, this highlights that the immunogenicity of RTX may be strongly associated to patient-related factors, in this case, the immunological and inflammatory status of the patients. Comparably, the major determinant of the development of ADAs to interferon-beta in MS was found to be high disease activity during treatment exposure [28].

As suggested by previous studies, systemic diseases seem to be more prone than RA in developing immunogenicity to RTX. In a retrospective analysis by Combier et al., having a systemic disease was a major factor associated to the presence of ADAs. The study though included only fifteen SLE patients, and five AAV, as the majority of systemic illnesses were represented by Sjögren's syndrome [12].

We observed that peripheral blood B cell counts at follow-up were higher in ADA-positive patients compared to ADA-negative, which contrast with a recent report [15] but is consistent with an aforementioned clinical trial [4]. However, if these higher B cell counts reflect a less efficient $B$ cell depletion or an accelerated repopulation, remains unclear.

Although the B cell counts could be influenced by diverse modalities of data collection and power of the studies, this might suggest that ADAs could influence the pharmacokinetic and pharmacodynamic of the drug leading to pharmacologic abrogation and reduced therapeutic exposure. Despite enrolling a higher number of patients compared to previous studies, the reduced availability of data concerning the peripheral B cell counts resulted in a lack of power for our study. Therefore, we were not fully able to evaluate the association between ADA titres and B cell counts or depletion as a surrogate of RTX efficacy. 
In fact, we could not observe a strong and direct correlation between ADA titres and B cell counts. This may also be explained on a pharmacological level. ADAs above a certain threshold, which exceeds the amount of circulating drug, may saturate the available drug and form immune-complexes but not impact further on the reduction of B cell count which follows RTX action.

Moreover, although we observed a difference in peripheral B cell counts between ADA-positive and ADAnegative patients, we could not confirm this difference when we further examined the rate of achievement of $B$ cell depletion. Furthermore, it is important to note that, given we evaluated patients in this study after the first exposure to RTX, the interpretation of the effect of ADAs on $B$ cell count following immunization to the drug is incomplete. Indeed, the real impact on B cell depletion efficiency might be expected upon retreatment.

In line with previous observations, we could confirm that the immunogenicity of RTX was not influenced by the concomitant administration of immunosuppressants such as intravenous corticosteroids and cyclophosphamide. This contrasts to other autoimmune diseases such as RA, where the concomitant treatment with methotrexate has been shown to be protective against, or at least delaying, the development of ADAs against infliximab, another chimeric monoclonal antibody [29].

Of interest is the striking difference between the ADA frequencies in SLE and AAV, as these might reflect different disease associated ADA risk factors in terms of RTX immunogenicity. On an immunological level, although both autoimmune in nature, the two diseases are characterized by different levels and quality of $\mathrm{B}$ cell reactivity and the propensity to respond to biotherapeutics with ADA formation in SLE compared to AAV, might be related to a more profound disruption of $B$ cell tolerance [30, 31].

This difference might also be implicated in the fact that we also found that a small portion of SLE samples, although naïve to RTX, were ADA-positive at low titre $(<2 \mathrm{AU} / \mathrm{mL})$, which although a rare event was still higher than in AAV. These ADAs were still above the diseasespecific cut-point established according to internationally established recommendations [32] and suggest the presence of naturally occurring antibodies, which in turn, should reflect the intrinsic B cell hyperreactivity typical of SLE and the presence of a remarkably broad antibody repertoire.

With respect to safety, our retrospective analysis of the clinical records allowed us to trace immediate and lateonset reactions in the ADA-positive patients who were retreated with RTX.

However, while the recording of immediate reactions was performed directly at the hospital, those of the late-onset reactions mostly relied on patients reporting such reactions afterwards.

Therefore, the possibility arises that our study might underestimate the true occurrence of serum sickness reactions.

We could also observe that ADA-positive patients who received a second course of RTX for early nephritic flares, did not respond in general, and all had higher ADA titres, though the low numbers in our study do not allow to make generalizations.

Taken together, our findings suggest the necessity of an increased awareness for the immunogenic consequences of RTX administration in SLE, while such phenomenon appears not to be of greater relevance for AAV. Based on our findings, we believe screening for ADA should be implemented following RTX treatment, in particular in younger patients with lupus nephritis as a leading manifestation. In such patients, retreatment should be considered carefully, both for possible lack of efficacy and for safety reasons, and alternative B cell depletive drugs could be considered in the case of ADA positivity. Further investigations are also needed to evaluate the rate of immunogenicity of RTX biosimilars in the context of SLE.

Further research should also address the question on whether RTX doses currently used might play a role in determining the immunogenicity of the drug and whether repeated treatments at regular intervals may affect and mitigate it. Indeed, in our cohort, given the large timespan to retreatment, possible effects of repeated exposure to RTX in terms of tolerization could not be investigated. A deeper comprehension of the immunological and clinical impact of ADA in RTX treated SLE patients warrants the conduction of longitudinal studies in order to better define which patients should stop RTX and/or be switched to alternative anti-CD20 agents.

\section{Conclusions}

To conclude, our study presents the result of a large real-life cohort of SLE patients in which we could demonstrate a high occurrence of ADA to RTX after the first exposure, highlighting how the patients more in need of RTX are also the ones more affected by the phenomenon of immunogenicity. In comparison to AAV, the reason for immunogenicity may be related to intrinsic immunological features of SLE. We believe that our findings should encourage further studies and the implementation of screening of SLE patients in the clinical routine.

\section{Abbreviations}

ADAs/ADA: Anti-drug antibodies; RTX: Rituximab; SLE: Systemic lupus erythematosus; AAV: ANCA-associated vasculitis; ANCA: Anti-neutrophil cytoplasmic antibodies; GPA: Granulomatosis with polyangiitis; MPA: Microscopic 
polyangiitis; EGPA: Eosinophilic granulomatosis with polyangiitis; RA: Rheumatoid arthritis; MS: Multiple sclerosis; SLEDAI-2 K: Systemic Lupus Erythematosus Disease Activity Index-2000; BVAS: Birmingham Vasculitis Activity Score; ACR : American College of Rheumatology; EMA: European Medicine Agency; $\mathrm{NIH}$ : National Institutes of Health; DMARDs: Disease-modifying anti-rheumatic drugs; AZA: Azathioprine; MMF: Mycophenolate mofetil; MTX: Methotrexate; CS: Corticosteroids; IR: Infusion reactions; ECL: Electrochemiluminescence (immunoassay); MSD: Meso Scale Discovery (platform); AU/mL: Arbitrary unit per millilitre; Anti-dsDNA: Anti-double strand-DNA antibodies; U-ACR: Urinealbumin to creatinine ratio; BCD: B cell depletion; CD19: Cluster of differentiation-19; CD20: Cluster of differentiation-20; HACA: Human anti-chimeric antibodies; IQR: Interquartile range; CNS: Central nervous system; N: Number.

\section{Acknowledgements}

We would like to thank Anna Mattson, Gloria Rostwall and Julia Norrko for the support with the laboratory procedures, sample handling and retrieval.

\section{Authors' contributions}

Francesca Faustini, Nicky Dunn, Iva Gunnarsson and Anna Fogdell Hahn designed and planned the study. Francesca Faustini and Iva Gunnarsson handled the selection of suitable patients for the study. Francesca Faustini arranged the collection of clinical data, performed data analysis (statistics). Nicky Dunn, Nastya Kharlamova, Malin Ryner handled the collection of laboratory data and conducted laboratory analysis concerning ADA determination. Francesca Faustini and Nicky Dunn drafted the manuscript. Iva Gunnarsson, Vivianne Malmström and Anna Fogdell-Hahn contributed to manuscript preparation. All the authors contributed to data interpretation and manuscript revision. The author(s) read and approved the final manuscript.

\section{Funding}

The study was financed by Region Stockholm by the ALF (Avtal för Läkarutbildning och Forskning), The Swedish Rheumatism Association, King Gustaf V's 80-year Foundation; Ingegerd Johansson's Fund, Karolinska Institutet Foundations, and the Swedish Kidney Foundation and Vinnova/Swelife (grant 2017-04936). Open access funding provided by Karolinska Institute.

\section{Availability of data and materials}

Data collection has been conducted in accordance to local regulation. In the original dataset, data are registered anonymously. The authors declare their availability in providing data if requested by the referees or the editorial team of the journal.

\section{Declarations}

Ethics approval and consent to participate

The study has been conducted in conformity to the approval of the Stockholm Region Ethic Committee (diary numbers 2001-152; 2008/1143-31; 2010/259-32).

\section{Consent for publication}

All authors have read the final draft of the article, and approve its submission for publication.

\section{Competing interests}

Anna Fogdell-Hahn: honoraria, lectures, advisory, boards, unrestricted research grants, Biogenldec, Pfizer, Roche, Johnson\&Johnson and Merck.The other authors have no competing interests to disclose.

\section{Author details}

${ }^{1}$ Department of Medicine Solna, Division of Rheumatology, Karolinska Institutet, Stockholm, Sweden. ${ }^{2}$ Unit of Rheumatology, Karolinska University Hospital, Stockholm, Sweden. ${ }^{3}$ Department of Clinical Neuroscience, Karolinska Institutet, Stockholm, Sweden. ${ }^{4}$ Center for Molecular Medicine, Stockholm, Sweden. ${ }^{5}$ Department of Health, Medicine and Caring Sciences, Linköping University, Linköping, Sweden. ${ }^{6}$ University Hospital and Department of Renal Medicine, Karolinska University Hospital and CLINTEC Karolinska Institutet, Stockholm, Sweden.

Received: 29 March 2021 Accepted: 23 July 2021

Published online: 13 August 2021

\section{References}

1. Maloney DG, Grillo-Lopez AJ, White CA, Bodkin D, Schilder RJ, Neidhart JA, Janakiraman N, Foon KA, Liles TM, Dallaire BK, et al. IDEC-C2B8 (Rituxi$\mathrm{mab}$ ) anti-CD20 monoclonal antibody therapy in patients with relapsed low-grade non-Hodgkin's lymphoma. Blood. 1997;90(6):2188-95.

2. Emery P, Fleischmann R, Filipowicz-Sosnowska A, Schechtman J, Szczepanski L, Kavanaugh A, Racewicz AJ, van Vollenhoven RF, Li NF, Agarwal $S$, et al. The efficacy and safety of rituximab in patients with active rheumatoid arthritis despite methotrexate treatment: results of a phase IIB randomized, double-blind, placebo-controlled, dose-ranging trial. Arthritis Rheum. 2006;54(5):1390-400.

3. Stone JH, Merkel PA, Spiera R, Seo P, Langford CA, Hoffman GS, Kallenberg CG, St Clair EW, Turkiewicz A, Tchao NK, et al. Rituximab versus cyclophosphamide for ANCA-associated vasculitis. N Engl J Med. 2010;363(3):221-32.

4. Looney RJ, Anolik JH, Campbell D, Felgar RE, Young F, Arend LJ, Sloand JA, Rosenblatt J, Sanz I. B cell depletion as a novel treatment for systemic lupus erythematosus: a phase I/II dose-escalation trial of rituximab. Arthritis Rheum. 2004;50(8):2580-9.

5. Hauser SL, Waubant E, Arnold DL, Vollmer T, Antel J, Fox RJ, Bar-Or A, Panzara M, Sarkar N, Agarwal S, et al. B-cell depletion with rituximab in relapsing-remitting multiple sclerosis. N Engl J Med. 2008;358(7):676-88.

6. Merrill JT, Neuwelt CM, Wallace DJ, Shanahan JC, Latinis KM, Oates JC, Utset TO, Gordon C, Isenberg DA, Hsieh HJ, et al. Efficacy and safety of rituximab in moderately-to-severely active systemic lupus erythematosus: the randomized, double-blind, phase II/III systemic lupus erythematosus evaluation of rituximab trial. Arthritis Rheum. 2010;62(1):222-33.

7. Rovin BH, Furie R, Latinis K, Looney RJ, Fervenza FC, Sanchez-Guerrero J, Maciuca R, Zhang D, Garg JP, Brunetta P, et al. Efficacy and safety of rituximab in patients with active proliferative lupus nephritis: the Lupus Nephritis Assessment with Rituximab study. Arthritis Rheum. 2012;64(4):1215-26.

8. Fanouriakis A, Kostopoulou M, Alunno A, Aringer M, Bajema I, Boletis JN, Cervera R, Doria A, Gordon C, Govoni M, et al. 2019 update of the EULAR recommendations for the management of systemic lupus erythematosus. Ann Rheum Dis. 2019;78(6):736-45.

9. Fanouriakis A, Kostopoulou M, Cheema K, Anders HJ, Aringer M, Bajema I, Boletis J, Frangou E, Houssiau FA, Hollis J, et al. 2019 update of the Joint European League Against Rheumatism and European Renal AssociationEuropean Dialysis and Transplant Association (EULAR/ERA-EDTA) recommendations for the management of lupus nephritis. Ann Rheum Dis. 2020;79(6):713-23.

10. Strand V, Goncalves J, Isaacs JD. Immunogenicity of biologic agents in rheumatology. Nat Rev Rheumatol. 2021;17(2):81-97. https://doi.org/10. 1038/s41584-020-00540-8.

11. Hamze M, Meunier S, Karle A, Gdoura A, Goudet A, Szely N, Pallardy M, Carbonnel F, Spindeldreher S, Mariette X, et al. Characterization of CD4 T cell epitopes of infliximab and rituximab identified from healthy donors. Front Immunol. 2017:8:500.

12. Combier A, Nocturne G, Henry J, Belkhir R, Pavy S, Le Tiec C, Descamps E, Seror $\mathrm{R}$, Mariette $\mathrm{X}$. Immunization to rituximab is more frequent in systemic autoimmune diseases than in rheumatoid arthritis: ofatumumab as alternative therapy. Rheumatology (Oxford). 2020;59(6):1347-54.

13. Dunn N, Juto A, Ryner M, Manouchehrinia A, Piccoli L, Fink K, Piehl F, Fogdell-Hahn A. Rituximab in multiple sclerosis: frequency and clinical relevance of anti-drug antibodies. Mult Scler (Houndmills, Basingstoke, England). 2017. https://doi.org/10.1177/1352458517720044.

14. Md Yusof MY, Shaw D, El-Sherbiny YM, Dunn E, Rawstron AC, Emery P, Vital EM. Predicting and managing primary and secondary non-response to rituximab using B-cell biomarkers in systemic lupus erythematosus. Ann Rheum Dis. 2017;76(11):1829-36.

15. Wincup C, Menon M, Smith E, Schwartz A, Isenberg D, Jury EC, Mauri C, Consortium A. Presence of anti-rituximab antibodies predicts infusionrelated reactions in patients with systemic lupus erythematosus. Ann Rheum Dis. 2019;78(8):1140-2.

16. Smith KG, Jones RB, Burns SM, Jayne DR. Long-term comparison of rituximab treatment for refractory systemic lupus erythematosus and vasculitis: remission, relapse, and re-treatment. Arthritis Rheum. 2006;54(9):2970-82. 
17. Tan EM, Cohen AS, Fries JF, Masi AT, McShane DJ, Rothfield NF, Schaller JG, Talal N, Winchester RJ. The 1982 revised criteria for the classification of systemic lupus erythematosus. Arthritis Rheum. 1982;25(11):1271-7.

18. Watts R, Lane S, Hanslik T, Hauser T, Hellmich B, Koldingsnes W, Mahr A, Segelmark M, Cohen-Tervaert JW, Scott D. Development and validation of a consensus methodology for the classification of the ANCA-associated vasculitides and polyarteritis nodosa for epidemiological studies. Ann Rheum Dis. 2007;66(2):222-7.

19. Knight A, Sundstrom $Y$, Borjesson O, Bruchfeld A, Malmstrom V, Gunnarsson I. Late-onset neutropenia after rituximab in ANCA-associated vasculitis. Scand J Rheumatol. 2016;45(5):404-7.

20. Gladman DD, Ibanez D, Urowitz MB. Systemic lupus erythematosus disease activity index 2000. J Rheumatol. 2002;29(2):288-91.

21. Mukhtyar C, Lee R, Brown D, Carruthers D, Dasgupta B, Dubey S, Flossmann O, Hall C, Hollywood J, Jayne D, et al. Modification and validation of the Birmingham Vasculitis Activity Score (version 3). Ann Rheum Dis. 2009;68(12):1827-32.

22. Gunnarsson I, Sundelin B, Jonsdottir T, Jacobson SH, Henriksson EW, van Vollenhoven RF. Histopathologic and clinical outcome of rituximab treatment in patients with cyclophosphamide-resistant proliferative lupus nephritis. Arthritis Rheum. 2007;56(4):1263-72.

23. Houssiau FA, Vasconcelos C, D'Cruz D, Sebastiani GD, Garrido Ed Ede R, Danieli MG, Abramovicz D, Blockmans D, Mathieu A, Direskeneli H, et al. Immunosuppressive therapy in lupus nephritis: the Euro-Lupus Nephritis Trial, a randomized trial of low-dose versus high-dose intravenous cyclophosphamide. Arthritis Rheum. 2002;46(8):2121-31.

24. Austin HA 3rd, Klippel JH, Balow JE, le Riche NG, Steinberg AD, Plotz PH, Decker JL. Therapy of lupus nephritis. Controlled trial of prednisone and cytotoxic drugs. New Engl J Med. 1986;314(10):614-9.

25. Bertsias GK, Tektonidou M, Amoura Z, Aringer M, Bajema I, Berden JH, Boletis J, Cervera R, Dorner T, Doria A, et al. Joint European League Against Rheumatism and European Renal Association-European Dialysis and Transplant Association (EULAR/ERA-EDTA) recommendations for the management of adult and paediatric lupus nephritis. Ann Rheum Dis. 2012;71(11):1771-82

26. Bayer G, Agier MS, Lioger B, Lepelley M, Zenut M, Lanoue MC, Maillot F, Jonville-Bera AP. Rituximab-induced serum sickness is more frequent in autoimmune diseases as compared to hematological malignancies: a French nationwide study. Eur J Intern Med. 2019;67:59-64.

27. Holmoy T, Fogdell-Hahn A, Svenningsson A. Serum sickness following rituximab therapy in multiple sclerosis. Neurol Clin Pract. 2019;9(6):519-21.

28. Dunn N, Fogdell-Hahn A, Hillert J, Spelman T. Long-term consequences of high titer neutralizing antibodies to interferon-beta in multiple sclerosis. Front Immunol. 2020;11:583560

29. Strand V, Balsa A, Al-Saleh J, Barile-Fabris L, Horiuchi T, Takeuchi T, Lula S, Hawes C, Kola B, Marshall L. Immunogenicity of biologics in chronic inflammatory diseases: a systematic review. BioDrugs. 2017;31(4):299-316.

30. Bashford-Rogers RJM, Bergamaschi L, McKinney EF, Pombal DC, Mescia F, Lee JC, Thomas DC, Flint SM, Kellam P, Jayne DRW, et al. Analysis of the $B$ cell receptor repertoire in six immune-mediated diseases. Nature. 2019;574(7776):122-6.

31. Dorner T, Giesecke C, Lipsky PE. Mechanisms of B cell autoimmunity in SLE. Arthritis Res Ther. 2011;13(5):243.

32. Gupta S, Devanarayan V, Finco D, Gunn GR 3rd, Kirshner S, Richards S, Rup B, Song A, Subramanyam M. Recommendations for the validation of cell-based assays used for the detection of neutralizing antibody immune responses elicited against biological therapeutics. J Pharm Biomed Anal. 2011;55(5):878-88.

\section{Publisher's Note}

Springer Nature remains neutral with regard to jurisdictional claims in published maps and institutional affiliations.
Ready to submit your research? Choose BMC and benefit from:

- fast, convenient online submission

- thorough peer review by experienced researchers in your field

- rapid publication on acceptance

- support for research data, including large and complex data types

- gold Open Access which fosters wider collaboration and increased citations

- maximum visibility for your research: over 100M website views per year

At BMC, research is always in progress.

Learn more biomedcentral.com/submissions 